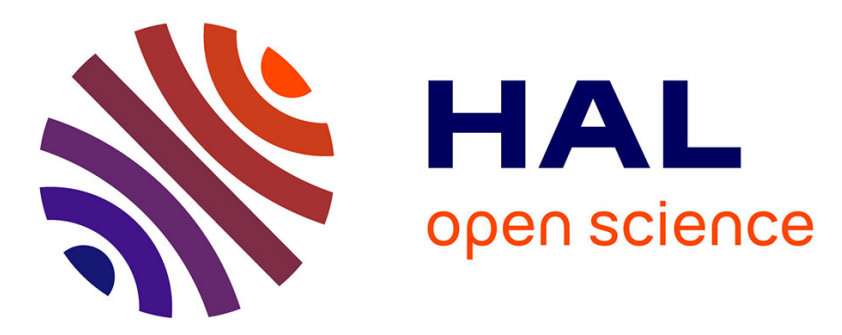

\title{
Bayesian Inference of Human Bone Sample Properties Using Ultrasonic Reflected Waves
}

\author{
Rémi Roncen, Zine El Abiddine Fellah, Erick Ogam
}

\section{To cite this version:}

Rémi Roncen, Zine El Abiddine Fellah, Erick Ogam. Bayesian Inference of Human Bone Sample Properties Using Ultrasonic Reflected Waves. Journal of the Acoustical Society of America, 2020, 148 (6), pp.3797-3808. 10.1121/10.0002878 . hal-03008582

\section{HAL Id: hal-03008582 \\ https://hal.science/hal-03008582}

Submitted on 2 Dec 2020

HAL is a multi-disciplinary open access archive for the deposit and dissemination of scientific research documents, whether they are published or not. The documents may come from teaching and research institutions in France or abroad, or from public or private research centers.
L'archive ouverte pluridisciplinaire $\mathbf{H A L}$, est destinée au dépôt et à la diffusion de documents scientifiques de niveau recherche, publiés ou non, émanant des établissements d'enseignement et de recherche français ou étrangers, des laboratoires publics ou privés. 


\title{
Bayesian Inference of Human Bone Sample Properties Using
}

\section{Ultrasonic Reflected Signals}

\author{
R. Roncen ${ }^{* 1}$, Z.E.A. Fellah ${ }^{2}$ and E. Ogam ${ }^{2}$ \\ ${ }^{1}$ ONERA /Département Multi-Physique pour l'Énergétique, Université de Toulouse, F-31055, Toulouse, France \\ ${ }^{2}$ Laboratoire de Mécanique et d'Acoustique, Centre National de la Recherche Scientifique, Unité Mixte de \\ Recherche 7031, Aix-Marseille Université, Centrale Marseille, F-13402 Marseille Cedex 20, France
}

\begin{abstract}
The non-intrusiveness and low cost of ultrasonic interrogation is motivating the development of new means towards the detection of osteoporosis and other bone deficiencies. Bone is a porous media saturated with a viscous fluid, and could thus be well characterized by the Biot model. The main purpose of this work is to present an in vitro methodology for the identification of the properties and structural parameters of the bone, adopting a statistical Bayesian inference technique using ultrasonic reflected signals at normal incidence. This approach allows the retrieval of some important parameters characterizing the bone structure and associated uncertainties, such as the porosity and bulk modulus. The method was applied to seven samples of bone extracted from a femoral head, immersed in water and exposed to ultrasonic signals with a center frequency of $\approx 500 \mathrm{kHz}$. For all seven samples, signals at different sites were acquired to check the method robustness.
\end{abstract}

\section{Introduction}

Osteoporosis affects bone density and microstructure [1, reducing bone quality and increasing the risk of fractures. The bone mass density (BMD) can be evaluated by X-Ray absorptiometry, but the BMD alone does not fully account for fracture risks [2]. There is thus a need to refine the characterization of bones, to help better understand the onset of bone aging and deterioration.

\footnotetext{
${ }^{*}$ Corresponding author: remi.roncen@onera.fr
} 
To avoid the invasive treatments that current radiographic or neutron activation technologies require for bone analysis, quantitative ultrasound assessments have been considered for more than 30 years [3-16] (for an osteoporosis-focused review, see Refs. 2, 17]). The main rationale for these studies is that an ultrasonic wave interacts differently between a normal and an osteoporotic bone.

Biot's theory links the microstructural and mechanical properties of poroelastic media to the behavior of waves propagating in them. While initially dedicated to geology, Biot's theory has received a lot of attention for its application to ultrasound propagation in bone [4, 14, 18, 22. Using the Biot model allows one to relate the microstructural and mechanical properties of the bone to its acoustic response. It then becomes possible to attempt an inverse problem, where the observation of a certain acoustic quantity (here an ultrasonic reflected signal) is used to infer the values of certain parameters of interest. This was done previously with the Biot model, using impedance tube measurements [23, reflected and transmitted ultrasonic waves [24] and, in our previous work, using transmitted ultrasonic waves only [25]. In the aforementioned references, the ill-posedness of the inverse problem was emphasized by the authors: if not enough prior information is available, different solutions to the inverse problems can be found.

While reflected waves have already been used in previous work for in vivo and in vitro ultrasonic interrogation of bone [10 12, 15, 26-28], only the diffusive properties of the waves were used (ultrasound backscatter). The present work looks at this problem under the scope of propagation properties of waves within a bone sample, with different physical phenomena at play.

Ultrasonic reflected waves are considered in this work at almost normal incidence on porous samples immersed in water. The reason behind this choice of studying only the reflected waves is that in some cases, the observation of a transmitted wave is made difficult due to the high dissipation of waves within the bone samples. When a wave encounters a discontinuity in its propagation medium, part of the wave is reflected back, while the rest is transmitted through the discontinuity. The signal measured in front of the material, coined reflected wave, is thus composed of a first reflected wave that has not traveled within the material, and of a succession of waves that have made it to the other end of the sample before coming back. It was shown for rigid porous material that even with only the first reflected wave, some information could be found on the porosity and tortuosity of the material [29 32]. Thus, even in the case of large samples or a very strong attenuation within the material (where no transmitted wave can be measured), some information might still be retrieved using the first reflected wave, as opposed to the transmission case.

A Bayesian inference approach is performed to update our state of belief (in the form of probability 
density functions) on the model parameters, which consist of the classical Biot parameters (density, Young's modulus, Poisson's ratio, etc.) and the intrinsic microstructure properties of the Horoshenkov model [33]. The main reason for the use of the Horoshenkov model in the present work is the need for a set of identified parameters that are easily understandable by the medical community (i.e., the mean pore radius is potentially clearer than the viscous characteristic length of the Johnson-Koplik-Dashen (JKD) pore model [34, often used in the acoustic community).

The study is organized as follows. The acoustical modeling, an extension of the Biot model to account for visco-inertial dissipation within the pore spaces, is recalled in Sec. [II The experimental configuration used to obtain the ultrasonic reflected signals and the statistical Bayesian inference problem are succinctly presented in Sec. III] In Sec. IV] the inference method is first applied on a synthetical test signal obtained via the modeling of Sec. II and artificially polluted by a random Gaussian noise to represent uncertainties in the measurement. The inference method is then applied on the experimental measurements of different bone samples extracted from different femoral heads. A conclusion is drawn in Sec. $\mathrm{V}$

\section{Acoustical model}

Bone is a porous material in which the fluid phase (composed of blood and marrow, i.e., a viscoelastic fluid) interacts with a solid phase (calcified tissue, with a complex microstructure that is linked to bone physical strength).

\section{A Biot model for the coupling between fluid and solid phases}

Biot's alternative formulation [35] is used in this work, including the additional visco-inertial dissipation that occurs within the pores. The following is similar to the approach detailed in Ref [24] and in our earlier work [25], and only differs in the definition of the semi-phenomenological pore model that is used (Horoshenkov model instead of Johnson model).

Equations are written in the frequency domain $\left(e^{+\mathrm{j} \omega t}\right.$ convention). The wavelength is assumed large compared with the size of the pores. Biot's coupling equations are given as

$$
\begin{gathered}
\omega^{2} \rho_{f} \boldsymbol{w}+\omega^{2} \rho \boldsymbol{u}=-\Delta \cdot \boldsymbol{\sigma}, \\
\omega^{2} \rho_{f} \boldsymbol{u}+\omega^{2} \tilde{\rho}_{\mathrm{eq}} \boldsymbol{w}=\Delta \cdot p,
\end{gathered}
$$

with $p$ the pressure field, $\boldsymbol{\sigma}$ the total stress tensor, $\boldsymbol{u}$ the solid phase displacement field, $\boldsymbol{w}$ the relative 
displacement field between the phases: $\boldsymbol{w}=\phi(\boldsymbol{U}-\boldsymbol{u})$ with $\boldsymbol{U}$ the fluid phase displacement field and $\phi$ the porosity. The angular frequency writes $\omega=2 \pi f$, with $f$ the frequency in $\mathrm{Hz} ; \rho_{f}$ is the ambient fluid density and $\rho=(1-\phi) \rho_{s}+\phi \rho_{f}$ is the density of the bulk medium, with $\rho_{s}$ the density of the calcified tissue. The equivalent density of the fluid phase $\tilde{\rho}_{\text {eq }}$ is a complex frequency dependent quantity that takes into account visco-inertial effects within the fluid phase inside the pores, and is defined in Sec. B.

The total stress tensor $\boldsymbol{\sigma}$ and the pressure field $p$ are related via the expressions

$$
\begin{aligned}
& \boldsymbol{\sigma}=2 N \boldsymbol{\epsilon}+\left(\lambda_{c} \xi-\alpha_{B} M \zeta\right) \mathbb{I}, \\
& p=M\left(\zeta-\alpha_{B} \xi\right)
\end{aligned}
$$

where $N$ is the shear modulus, $\boldsymbol{\epsilon}=\frac{1}{2}\left(\nabla \boldsymbol{u}+(\nabla \boldsymbol{u})^{T}\right)$ is the strain tensor, $\mathbb{I}$ is the identity matrix, $\xi=\nabla \cdot \boldsymbol{u}$ and $\zeta=-\nabla \cdot \boldsymbol{w}$. The Biot-Willis coefficient $\alpha_{B}$ is defined by

$$
\alpha_{B}=1-\frac{K_{b}}{K_{s}}
$$

with $K_{b}$ the bulk modulus of the porous frame and $K_{s}$ the bulk modulus of the solid constituent of the material. The shear modulus $N$, the Young's modulus and Poisson's ratio of the solid $E_{s}, \nu_{s}$ and the Young's modulus and Poisson's ratio of the porous frame $E_{b}, \nu_{b}$ are related as

$$
K_{s}=\frac{E_{s}}{3\left(1-2 \nu_{s}\right)}, \quad K_{b}=\frac{E_{b}}{3\left(1-2 \nu_{b}\right)}, \quad N=\frac{E_{b}}{2\left(1+\nu_{b}\right)} .
$$

The parameters $E_{s}$ and $\nu_{s}$ are non identifiable in this experiment, as they define a single parameter $K_{s}$, which is the one inferred in practice.

The elastic parameter $\lambda_{c}$ of Eq. 2 is defined as

$$
\lambda_{c}=\lambda+\alpha_{B}^{2} M
$$

where $\lambda$ is the first Lamé's coefficient of the elastic frame, and

$$
M=\left[\alpha_{B}+\left(\frac{K_{s}}{K_{f}}-1\right) \phi\right]^{-1} K_{s}
$$

with $K_{f}$ the bulk modulus of the fluid.

To take into account additional viscoelastic damping effects, a loss factor parameter $\xi_{s}$ (resp. $\xi_{b}$ ) is defined for the solid part of the material (resp. for the bulk). Structural parameters are updated as

$$
\begin{gathered}
K_{s} \rightarrow K_{s}\left(1+\mathrm{j} \xi_{s}\right), \\
E_{b} \rightarrow E_{b}\left(1+\mathrm{j} \xi_{b}\right), \\
N \rightarrow N\left(1+\mathrm{j} \xi_{b}\right) .
\end{gathered}
$$


The state vector formalism used in Refs. [24, 25] is used in this work, following the theory of Refs. [36, 37]. The initial problem of Eq. 1 is expressed in the physical space of the fluid and solid phases of the poroelastic material (the six unknowns are physical quantities: displacement, pressure, stress tensor). The problem can be recast in the eigenspace, where the waves amplitudes are the new unknowns. In poroelastic materials, three types of waves can propagate: two compression waves and one shear wave. Expressing the problem in terms of waves propagating in a direction or the other (thus totaling six unknown waves) allows for the removal of spurious evanescent terms in the formulation. This removes the problem of instability at high frequencies, encountered with the transfer matrix method, as shown in Ref [37]. The reflection coefficient $\mathcal{R}(\omega)$ is found after the solving of a linear system expressed with a sparse matrix containing the information of all frequencies simultaneously [24]. The reflection coefficient here contains all the contributions of the successive incoming reflected waves altogether. In this work, the frequency domain signals (reflection coefficients) are used for the identification procedure of the material parameters. While in some cases, working with the time domain signals can prove useful during the inference process (manual detection of the successive transmitted/reflected waves), it is also more computationally intensive due to the additional transformations between frequency and time domain at each iteration.

\section{B Semi-phenomenological model of Horoshenkov for the viscous dissipation within the fluid phase}

Bone samples are composed of pores whose shape and size obey a certain statistical distribution. The pore size distribution might be of importance, as an additional marker of bone health. In order to infer this parameter distribution from acoustic measurement, an adequate model has to be used. In the present work, the viscous dissipation in the pores is accounted for by the Horoshenkov model [33, based on the rational (Padé) approximation approach [38] and on the hypothesis that the pore size follows a log-normal distribution and that the pores have a circular shape. While the log-normal distribution hypothesis is typical of porous materials, and seems to hold for bone samples in the case of cancellous bones (as suggested in Ref. [39, Figs. 2-4]), the circular shape hypothesis might not hold as well as the former does. The influence of the shape parameters in the Horoshenkov model can, however, be neglected in a first approximation. A future study could try to infer these shape parameters as well, with the additional difficulty that an increase in the number of parameters could lead to a more ill-posed problem.

In the case where the above approximations are valid, the Horoshenkov model for viscous dissipation 
writes

$$
\tilde{\rho}_{\mathrm{eq}}=\frac{\rho_{f}}{\phi} \tilde{\alpha}(\omega)
$$

where the dynamic tortuosity $\tilde{\alpha}$ is defined as

$$
\tilde{\alpha}(\omega)=\alpha_{\infty}\left[1+\frac{1}{j \bar{\omega}} \tilde{F}(\omega)\right]
$$

with

$$
\begin{gathered}
\tilde{F}(\omega)=1-P+P \sqrt{1+\frac{M_{2}}{2 P^{2}} \mathrm{j} \bar{\omega}}, \quad \bar{\omega}=\omega \rho_{f} \frac{k_{0} \alpha_{\infty}}{\mu \phi}, \\
P=4 \frac{M_{2}}{2 \beta_{P} \phi \Lambda^{2}}, \quad M_{2}=8 \frac{k_{0} \alpha_{\infty}}{\phi \Lambda^{2}} .
\end{gathered}
$$

The parameters used in this model are the classical JKD ones [34, and relate to the microstructure of the porous sample: porosity $\phi$, tortuosity $\alpha_{\infty}$, permeability $k_{0}$ and characteristic viscous length $\Lambda$. Using the hypotheses of a log-normal pore distribution and a circular pore shape, it is possible to use only three parameters, namely the porosity $\phi$, mean pore size $\bar{s}$ and pore size standard deviation $\sigma_{s}$ (normalized by $\log (\bar{s}))$, instead of four. The relationships between JKD and Horoshenkov model parameters are [40, 41]

$$
\begin{aligned}
\phi & =\phi, \\
\alpha_{\infty} & =e^{4\left(\sigma_{s} \log 2\right)^{2}}, \\
k_{0} & =\frac{\bar{s}^{2} \phi}{8 \alpha_{\infty}} e^{-6\left(\sigma_{s} \log 2\right)^{2}}, \\
\Lambda & =\bar{s} e^{-\frac{5}{2}\left(\sigma_{s} \log 2\right)^{2}}, \\
\beta_{P} & =\frac{4}{3} e^{4\left(\sigma_{s} \log 2\right)^{2}} .
\end{aligned}
$$

The main drawback of using the Horoshenkov model in the present study is the lack of information regarding the true pore shape, here assumed to be circular. However, assuming a pore size distribution seems more appropriate to this kind of analysis than simply assuming a single value for the pore size. In the present work, the pore size distribution parameters $\bar{s}$ and $\sigma_{s}$ are themselves considered as random variables during the inference process, similarly to the other variables.

\section{Identification method}

This section presents the different elements required to perform the identification of the bone parameters of interest, using an inverse method (statistical inference). The experimental apparatus used to measure the 


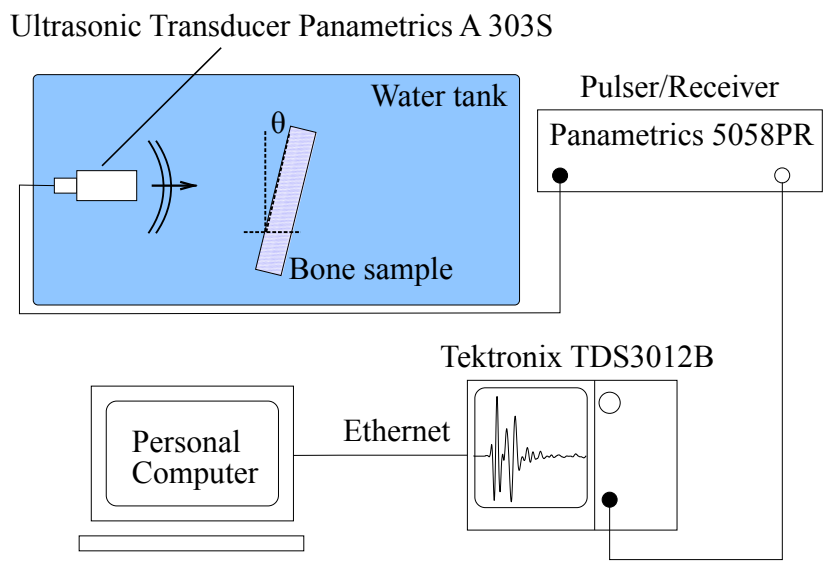

Figure 1: Experimental setup for ultrasonic measurements

reflected waves is first given, followed by a succinct summary of the Bayesian inference approach used to find the posterior density functions of the parameters.

\section{A Experimental apparatus}

Experiments are performed in water using one Panametrics A 303S plane piezoelectric transducer of diameter $1 \mathrm{~cm}$, with a $500 \mathrm{kHz}$ central frequency. Pulses of $400 \mathrm{~V}$ are provided by a $5058 \mathrm{PR}$ Panametrics pulser/receiver, amplified to $90 \mathrm{~dB}$, filtered above $10 \mathrm{MHz}$ and averaged over 1000 iterations. A schematic of the experiment is shown in Fig. 1 The size of the ultrasound beam is small compared with the size of the specimens. The liquid initially in the pore space (blood and marrow) has been removed from the bone sample and substituted by water. The bone samples, machined from the cancellous parts of a femoral head and neck of different human specimens, are constantly immersed in liquid to prevent them from drying, which can alter their properties [42]. The angle of incidence of the incident wave $\theta$ (displayed in Fig. 1) is considered small but unknown.

For each test, the sample is first replaced by a totally reflecting metal plate, and the reflected wave is recorded and is considered the reference signal. Then, the sample is placed and a new reflected wave is measured by the transducer and recorded. Transforming both signals in frequency domain and dividing the second one by the first one yields the reflection coefficient of the sample.

\section{B Identification method: Bayesian inference}

Within the Bayesian inference framework used in this work, all the model parameters are considered as random variables associated with probability density functions (pdf). These pdfs encode the information one 
has on the parameters [Chap. 843]. An experimental dataset $v^{\text {obs }}$ is observed (here a reflection coefficient), thus potentially increasing our state of knowledge on the variables of interest. $v^{\text {obs }}$ is the realization of a multivariate random variable $\Upsilon^{\text {obs }}$ (we separate the real and imaginary parts, assumed uncorrelated). The information on the (random variable) parameters $Q=(\phi, \bar{s}, \ldots)$, with realizations $q$, is encoded within the marginal posterior density $\pi\left(q \mid v^{\text {obs }}\right)$. This quantity of interest is written, using Bayes theorem,

$$
\pi\left(q \mid v^{\mathrm{obs}}\right)=\frac{\pi\left(v^{\mathrm{obs}} \mid q\right) \pi_{0}(q)}{\pi\left(v^{\mathrm{obs}}\right)}
$$

In the previous equation, $\mathcal{L}(q) \equiv \pi\left(v^{\text {obs }} \mid q\right)$ is the likelihood function, representing the probability of the experiment being observed, given a realization $q$ of model parameters; $\pi\left(v^{\text {obs }}\right)$ is an integral not calculated in practice because of the potentially high dimension of the parameter space; $\pi_{0}(q)$ is the prior probability and represents all the information obtained on $q$ before the new observation (i.e, one could weigh the sample to access its overall density $\rho$, thus providing bounds during the identification of $\phi$ and $\rho_{s}$ ). In this work, almost no previous knowledge on the bone parameters is used, so $\pi_{0}$ is a uniform distribution, whose bounds depend on the case considered.

Contrary to our previous work [25], frequency domain information is now used to build the likelihood. The rationale for this change is that staying in the frequency domain avoids the additional use of fast Fourier transformations for comparison with the measured time domain signals. When considering a Gaussian error representing the measurement uncertainties, the likelihood writes as the product of the likelihoods associated with both the real (subscript $\Re$ ) and imaginary parts (subscript $\Im$ ) of the reflection coefficient:

$$
\pi\left(v_{i}^{\mathrm{obs}} \mid q\right)=\pi_{\Re} \cdot \pi_{\Im}
$$

with

$$
\pi_{\Re}=\prod_{i=1}^{n} \frac{1}{\sqrt{2 \pi \sigma_{\Re, i}^{2}}} e^{-\left|\Re\left(v_{i}^{\mathrm{obs}}-R_{i}(q)\right)\right|^{2} / 2 \sigma_{\Re, i}^{2}}
$$

and a similar definition for $\pi_{\Im}$, where $n=50$ is the number of discrete frequencies where the reflection coefficient was measured, and $R_{i}(q)$ is the numerical reflection coefficient. The noise parameter $\sigma_{\Re, i}$ (resp. $\left.\sigma_{\Im, i}\right)$ is a standard deviation of the assumed Gaussian distribution of errors on the real part (resp. imaginary part). This parameter is included in the inference process, but a single value $\sigma$ is assumed at all frequencies and for both real and imaginary parts, since they have the same order of magnitude in most cases.

In contrast to what is described in Ref [24], only the reflection coefficient is used, not the transmission coefficient. The objective of this work is to evaluate the type of knowledge that can be extracted from this quantity only. The use of the Horoshenkov model also makes it possible to reduce the parameter space 
dimension by one, when compared with the Biot-JKD approach in the high frequency regime. If the frequency regime is arbitrary and the high-frequency limit cannot be used anymore, the Biot-JKD model makes use of between 4 and 5 parameters depending on whether the model improvements made by Pride [44] are used or not. By contrast, the Horoshenkov model still uses only 3 parameters. This reduction is made at the cost of a strong hypothesis, i.e., that of the pore size following a log-normal distribution.

To solve the problem and obtain $\pi\left(q \mid v^{\text {obs }}\right)$, a Markov Chain Monte Carlo strategy is used to explore the parameter space and sample from the target distribution. Similarly to our previous work [25, Sec. 4.2], the algorithm strategy developed in Ref. 45] is used, which performs well on multimodal distributions of dimensions $<20$.

\section{Results}

This sections presents two different kind of results. First, the model of Sec. II is used with known parameters to create a signal (reflection coefficient). This signal is then polluted by a noise, and the inference strategy of Sec. III is applied to evaluate the method capabilities and potential shortcomings. Then, the inference is performed on experimental signals obtained on seven different human femoral head samples.

\section{A Inference of a known synthetic material}

In order to evaluate the proposed inference strategy, a test case is developed. A set of known parameter values $q^{*}$ is chosen to feed the model of Sec. II and create a reflection coefficient between $200 \mathrm{kHz}$ and $700 \mathrm{kHz}$, which is the typical frequency range of our transducers. These parameters are close to the ones inferred later on in Sec. B. A random Gaussian noise of standard deviation $\sigma=5 \cdot 10^{-3}$ is then added to both the real and imaginary parts of the signal to artificially pollute it and represent the measurement uncertainties. The value of $\sigma$ corresponds to $3.2 \%$ of the maximum amplitude of the real part of the reflection coefficient, and $6.6 \%$ of the maximum amplitude of the imaginary part of the signal. To test the limits of the method, the prior bounds are all taken quite large, even when the property is expected to be well known or easily measured in practice (i.e., angle of incidence, sample thickness and ambient fluid properties). The bounds of the prior $q_{\min }$ and $q_{\max }$ are given in Table 1, where the true value $q^{*}$ is recalled and where the inference results are also given by their mean and standard deviation (st. dev). The posterior densities are displayed in Fig. 2 where the vertical dashed blue line represents the true value. The noisy signal used for the inference is shown on the right side in Fig 2 and where the 65\% (resp. 99.7\%) credibility intervals are given (dark and light 
gray zones, respectively). To evaluate the credibility intervals, a number of $N=10000$ samples from the Markov chains are used to calculate the reflection coefficient. The gray areas correspond to a plot around the calculated reflection coefficient mean value, with a distance from the mean equal to 1 (resp. 3) standard deviations.

Table 1: True values $q^{*}$, inferred values and prior bounds $q_{\min / \max }$ of the parameters for the synthetic test case. The symbol - means unitless

\begin{tabular}{|c|c|c|c|c|c|c|c|c|c|c|c|c|c|c|}
\hline Parameter & $\theta$ & $L$ & $\phi$ & $\bar{s}$ & $\sigma_{s}$ & $K_{s}$ & $\xi_{s}$ & $E_{b}$ & $\xi_{b}$ & $\nu_{b}$ & $\rho_{s}$ & $K_{f}$ & $\mu_{f}$ & $\rho_{f}$ \\
\hline Name & $\begin{array}{l}\frac{0}{00} \\
\underset{Z}{Z}\end{array}$ & 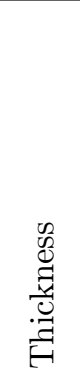 & 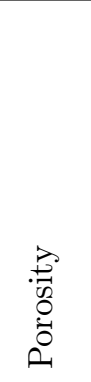 & 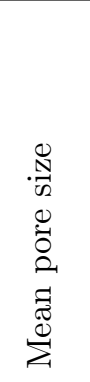 & 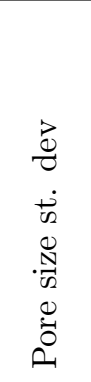 & 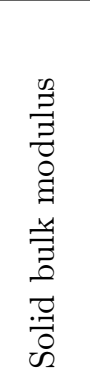 & 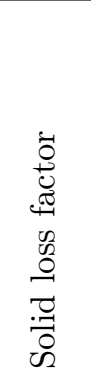 & 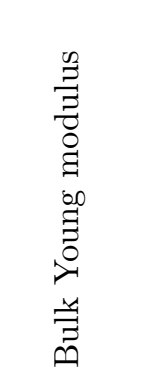 & 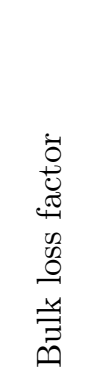 & 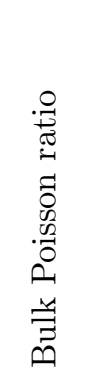 & 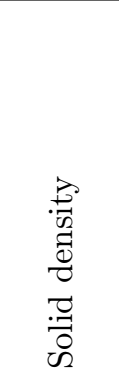 & 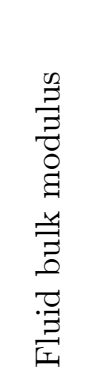 & 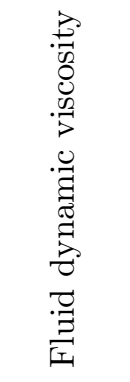 & 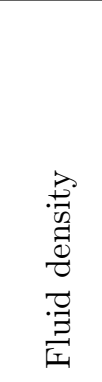 \\
\hline Unit & $\mathrm{rad}$ & $\mathrm{mm}$ & - & $\mu \mathrm{m}$ & - & $\mathrm{GPa}$ & - & $\mathrm{GPa}$ & - & - & $\mathrm{kg} / \mathrm{m}^{3}$ & $\mathrm{GPa}$ & $\mathrm{mPa} \cdot \mathrm{s}$ & $\mathrm{kg} / \mathrm{m}^{3}$ \\
\hline$q^{*}$ & 0.02 & 15 & 0.85 & 20 & 0.2 & 50 & 0.1 & 1.3 & 0.1 & 0.3 & 1.95 & 2.4 & 1 & 1 \\
\hline mean & -0.005 & 13.2 & 0.86 & 20.6 & 0.22 & 61.4 & 0.42 & 1.26 & 0.1 & 0.23 & 2.5 & 2.2 & 1.1 & 1.1 \\
\hline st. dev & 0.099 & 1.9 & 0.02 & 0.4 & 0.07 & 32.8 & 0.24 & 0.47 & 0.015 & 0.11 & 0.67 & 0.58 & 0.26 & 0.19 \\
\hline$q_{\min }$ & -0.2 & 5 & 0.4 & 0.1 & 0 & 1 & 0 & $0.001 K_{s}$ & 0 & 0.05 & 0.1 & 1.4 & 0.5 & 0.5 \\
\hline$q_{\max }$ & 0.2 & 25 & 0.99 & 1500 & 2 & 150 & 0.95 & $0.95 K_{s}$ & 0.95 & 0.49 & 5 & 3.4 & 1.5 & 1.5 \\
\hline
\end{tabular}

For all parameters, the posterior density function encompasses the true value. However, the uncertainty on the identified parameters can be quite large, relatively to the prior uncertainty. Notable cases are $K_{s}, \xi_{s}$, the ambient fluid parameters $\left(K_{f}, \mu_{f}, \rho_{f}\right)$ and the solid density $\rho_{s}$, which all seem to have a low sensitivity with respect to the likelihood function. This, we believe, is a theoretical limit of the present method.

In the inference performed on experimental signals, the focus will be placed on four parameters that can be well identified in theory and that could represent an interest to the medical field: $\phi, \bar{s}, \sigma_{s}, E_{b}$.

\section{B Inference of femoral heads}

Seven samples of different femoral heads were tested during this study. For each sample, between 2 and 5 reflected signals were acquired at different site locations, depending on the size of the sample. 

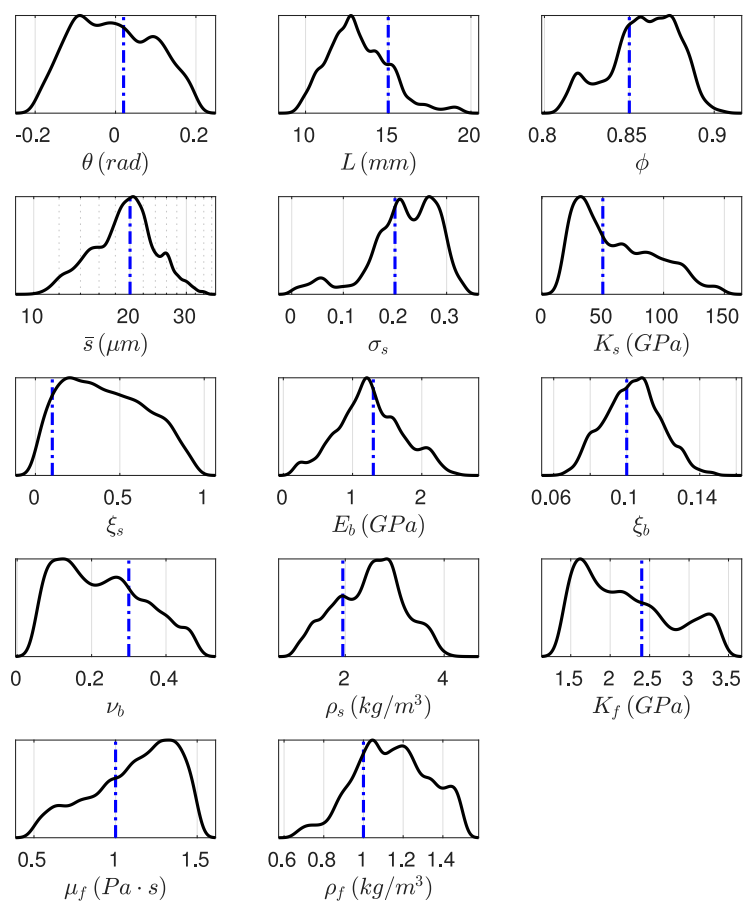

(a) (Color online). Probability density functions of identified parameters of the synthetic material. The dashed blue vertical line corresponds to the true value.

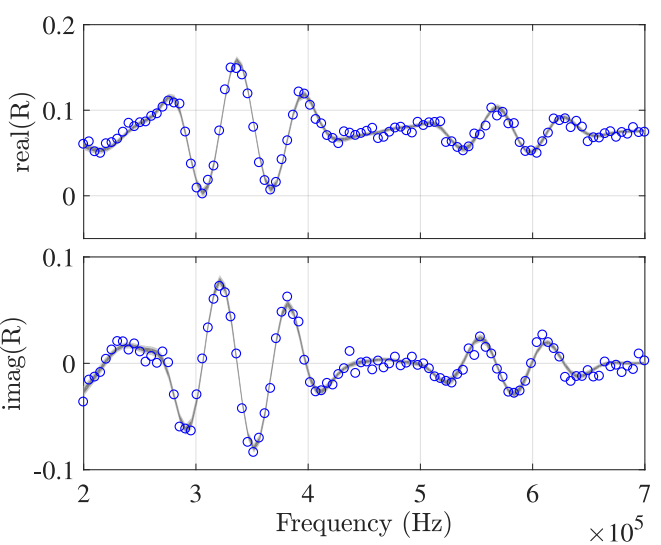

(b) (Color online). Real and imaginary parts of the teflection coefficient of the synthetic material. Blue markers correspond to the noisy data used during the inference. The gray areas correspond to the credibility intervals of the reconstructed signal using samples from the probability functions.

Figure 2: Inference results for the synthetic sample.

Using the test case of Sec. A it becomes clear that the proper identification of the fluid properties and of the solid density are not possible with the present method, due to their lack of sensitivity in the likelihood function. While the ambient fluid parameters are fairly well known, the solid density of bone doesn't have a fixed known value and can change in test subjects due to a condition called osteopenia. To avoid possible ill-posedness in the problem, as in Ref. [25], the prior of the solid density was constrained too.

The bounds of the prior are given in Table 2

Table 2: Prior bounds of the parameters for bone samples. The symbol - means unitless

\begin{tabular}{lcccccccccccccc}
\hline \hline Parameter & $\theta$ & $L$ & $\phi$ & $\bar{s}$ & $\sigma_{s}$ & $K_{s}$ & $\xi_{s}$ & $E_{b}$ & $\xi_{b}$ & $\nu_{b}$ & $\rho_{s}$ & $K_{f}$ & $\mu_{f}$ & $\rho_{f}$ \\
\hline Unit & $\mathrm{rad}$ & $\mathrm{mm}$ & - & $\mu \mathrm{m}$ & $/$ & $\mathrm{GPa}$ & - & $\mathrm{GPa}$ & - & - & $\mathrm{kg} / \mathrm{m}^{3}$ & $\mathrm{GPa}$ & $\mathrm{mPa} \cdot \mathrm{s}$ & $\mathrm{kg} / \mathrm{m}^{3}$ \\
\hline \hline$q_{\min }$ & -0.05 & $0.9 L_{i}^{*}$ & 0.4 & 1.0 & 0 & 5 & 0 & $0.001 K_{s}$ & 0 & 0.1 & 1.8 & 2.3 & 0.95 & 0.95 \\
$q_{\max }$ & 0.05 & $1.1 L_{i}^{*}$ & 0.99 & 100 & 2 & 150 & 0.9 & $0.9 K_{s}$ & 0.5 & 0.45 & 2.2 & 2.5 & 1.05 & 1.05 \\
\hline \hline
\end{tabular}


Table 3 summarizes the characteristics of the posterior probability density function (pdf) of each parameter, giving for each the mean and standard deviation. Although some distributions are not perfectly Gaussian, the mean and standard deviation have been chosen here because they remain simple and widespread indicators. When the non-Gaussianity is strong, the standard deviation can exceed the mean, suggesting the possibility of negative values, while in the actual Markov chain, no negative value is encountered. A special case occurs for sample 2, where a bi-modal distribution is obtained for $E_{b}$ for two out of the three tests that were performed. This indicates that for these signals, the model interprets both distinct modes (i.e., peak density) as likely candidates. More credit should be given to the first mode (lower value), due to its value being closer to what was found for other samples. In Table 3, the mean and standard deviation is given for both modes, as they are sufficiently separated.

The parameters $\rho_{s}, K_{f}, \mu_{f}$ and $\rho_{f}$ were, as expected, consistently displaying an almost uniform probability density covering the entirety of their prior support: $\rho_{s}=2.0 \pm 0.1 \mathrm{~kg} / \mathrm{m}^{3}, K_{f}=2.40 \pm 0.023 \mathrm{GPa}$, $\mu_{f}=1.0 \pm 0.02210^{-3} \mathrm{~N} \cdot \mathrm{s} / \mathrm{m}^{2}$ and $\rho_{f}=1.0 \pm 0.024 \mathrm{~kg} / \mathrm{m}^{3}$. Their values have been omitted from Table 3 for clarity. The values for the angle of incidence and the sample thickness were both well identified, with a pdf that was narrow compared with the original extent of the prior support. The parameters were omitted from the table for clarity.. The measured thicknesses $L_{i}^{*}$ of Table 2, with $i$ the sample number, were obtained by caliper measurements: $L_{1}^{*} \in[4.5-8.5] \mathrm{mm}, L_{2}^{*} \in[5.9-7.4] \mathrm{mm}, L_{3}^{*} \in[4.4-8.4] \mathrm{mm}, L_{4}^{*} \in[15-15.9] \mathrm{mm}$, $L_{5}^{*} \in[9.2-12.1] \mathrm{mm}, L_{6}^{*} \in[10.6-11.6] \mathrm{mm}, L_{7}^{*} \in[7.5-7.7] \mathrm{mm}$.

Using only reflected signals in the ultrasonic range for this study, we were not able to estimate all parameters accurately. For instance, the parameters related to the ambient fluid $\left(K_{f}, \mu_{f}, \rho_{f}\right)$ all display a distribution that spanned the entirety of the prior bounds that were set. However, the focus of this work was on the proof of identifiability of the parameters pertaining to the bone strength. Four of these parameters were consistently well identified, i.e., the porosity $\phi$, the pore mean size $\bar{s}$, the pore size standard deviation $\sigma_{s}$ and the porous bulk Young modulus $E_{b}$. Their pdfs are shown on the left side in Figs. $3[4|5| 6|7| 8 \mid 9$, where each line style or color corresponds to a given measurement on the same sample, and at a different location (note that a $\log$ scale is used for $\bar{s}$ ). Bone samples are known to display strong heterogeneities in both the trabecular and cortical regions. As the ultrasonic interrogation was performed at different positions over the bone sample each time, it is then not surprising to obtain different pdfs for each test. However, despite these differences in the pdfs for a given material sample, the values typically fall within a similar range. This tends to show that the problem is not too ill-posed: a variation in the data (the reflected wave signal) does not 
yield an arbitrarily high variation in the output (the pdfs). This is reassuring, given the goal of extending this method to in vivo measurements in the future.

Even when samples differ, their parameters exhibit similar values. Of particular importance, the frame Young modulus $E_{b}$ consistently displays a well defined pdf whose support is between 1 and $4 \mathrm{GPa}$, well within the range observed in direct measurements on wet cancellous bone [46, Fig. 3], albeit on the upper side of the values found in the literature. The porosities and mean pore size radii are equally well identified, and also display values within the range found in the literature on trabecular bones $(0.7-0.9$ for the porosity, and $1 \mu \mathrm{m}-1 \mathrm{~mm}$ for the pore size). In our previous study, ultrasonic waves transmitted in a femoral head sample were used. For sample M3 of Ref. 25] (not used in the present work), a porosity of $\phi=0.84$ and a viscous length of $\Lambda=40.5 \mu \mathrm{m}$ were found, which is equivalent to $\bar{s}=42.5 \mu \mathrm{m}$ with $\sigma_{s}=0.2$. A lower value of $\left|E_{b}\right|$ was also found $(0.71 \mathrm{GPa})$. Further studies will be required to explain why, in the present study, values of $E_{b}$ are consistently on the higher end of the spectrum.

In addition to the pdfs of the parameters, the experimental reflected signals are compared with the numerical realizations associated with samples of the pdfs (drawn directly from the Markov Chains so that the correct distributions are used). The time-domain fit between experimental and numerical signals is displayed on the right side in Figs 3445667869 , where the 65\% (resp. 99.7\%) credibility intervals are given (dark and light gray zones, respectively). The line style indicates the correspondence between experiments and the related identified pdf, in the left and right figures. To evaluate the credibility intervals on the time domain signals, a number of $N=10000$ samples from the Markov chains are used after each inference to calculate the reflection coefficients in the frequency domain. The signal is transformed back into time domain via an inverse FFT after multiplication with the incident signal. At each time step, the $N$ values of the signals are stored. The distribution of the output at each time step is assumed Gaussian, and the gray areas on the right side in Figs $3[4[5] 6 / 7 / 8 \mid 9$ correspond to a plot around the signal mean value, with a distance from the mean equal to 1 (resp. 3) standard deviations.

While the numerical signals are in quite good agreement with the experimental signals, a discrepancy is observed for some signals at later times: a stronger amplitude of the secondary oscillations at around $2.2 \cdot 10^{-5} \mathrm{~s}$ is displayed on the numerical signals (see e.g. Figs 78 ). The reason for this discrepancy is not immediately clear. It is possible that the heterogeneity of these samples participate in the acoustic response in an unexpected manner (i.e., not predictable by the model). 

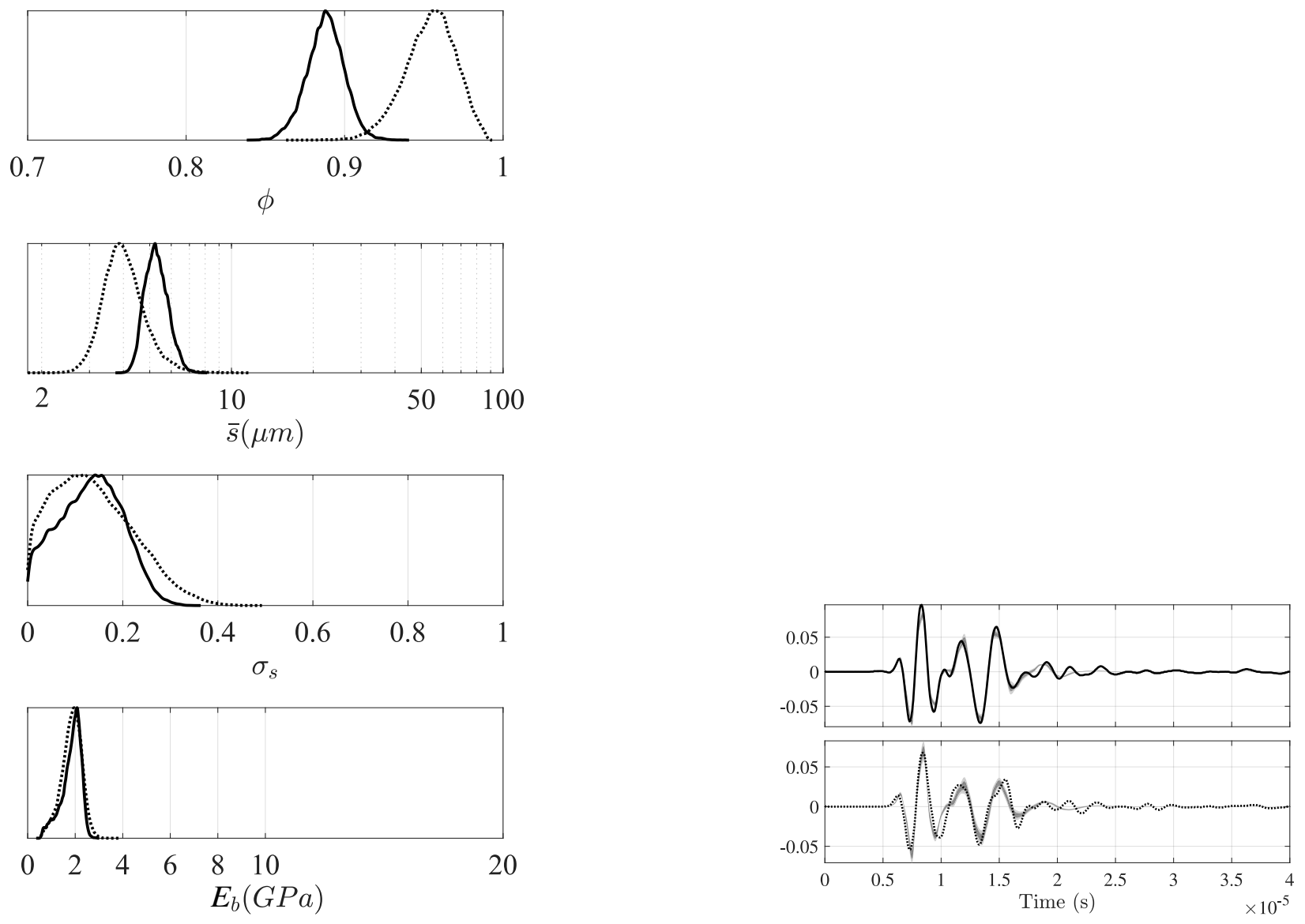

(a) Probability density functions of selected bone (b) Experiment vs fitted model comparison of time structural parameters for different measurements domain reflected signals on the bone sample.

Figure 3: Inference results for sample M1 

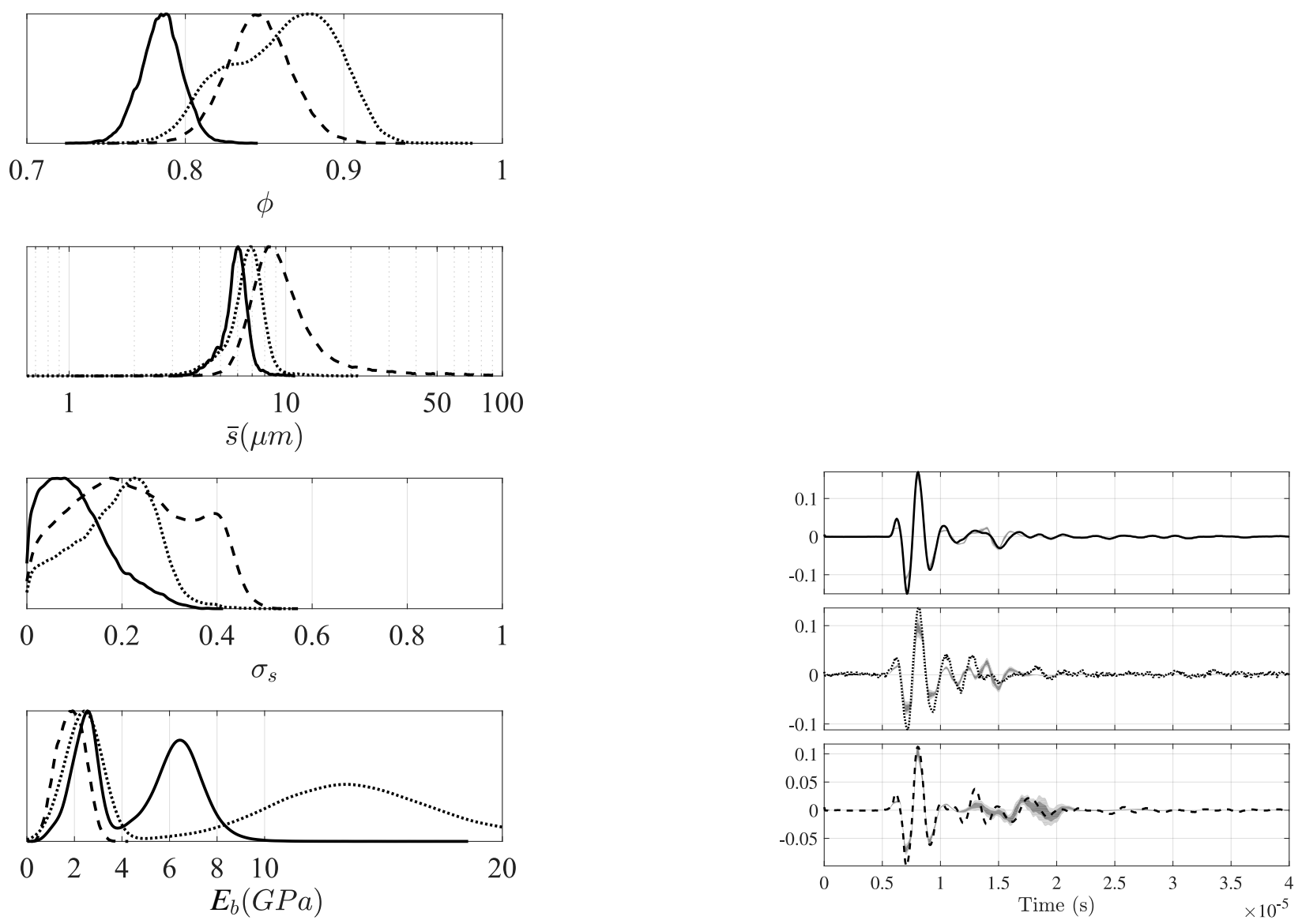

(a) Probability density functions of selected bone

(b) Experiment vs fitted model comparison of time structural parameters for different measurements domain reflected signals on the bone sample.

Figure 4: Inference results for sample M2 

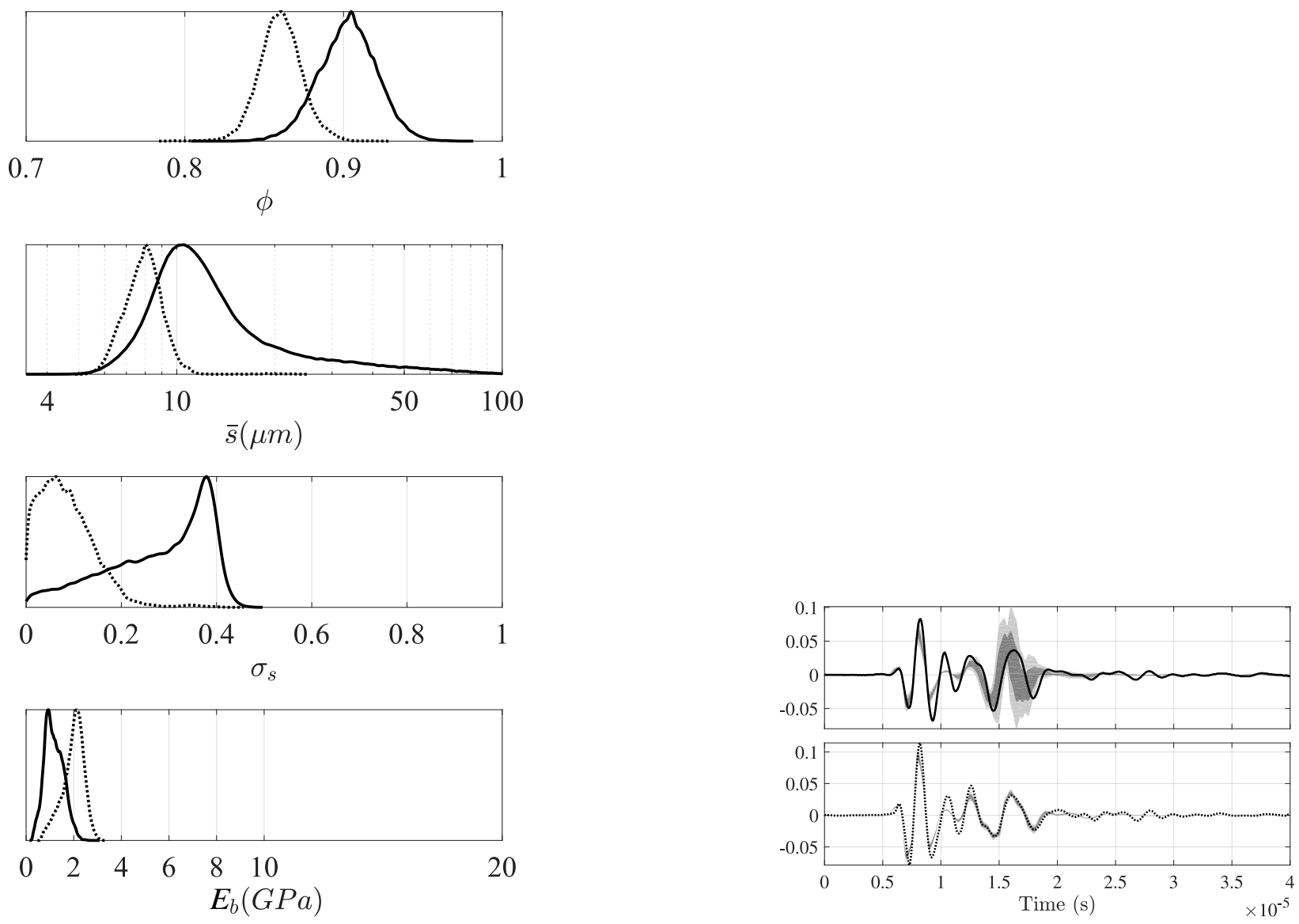

(a) Probability density functions of selected bone

(b) Experiment vs fitted model comparison of time structural parameters for different measurements domain reflected signals on the bone sample.

Figure 5: Inference results for sample M3 

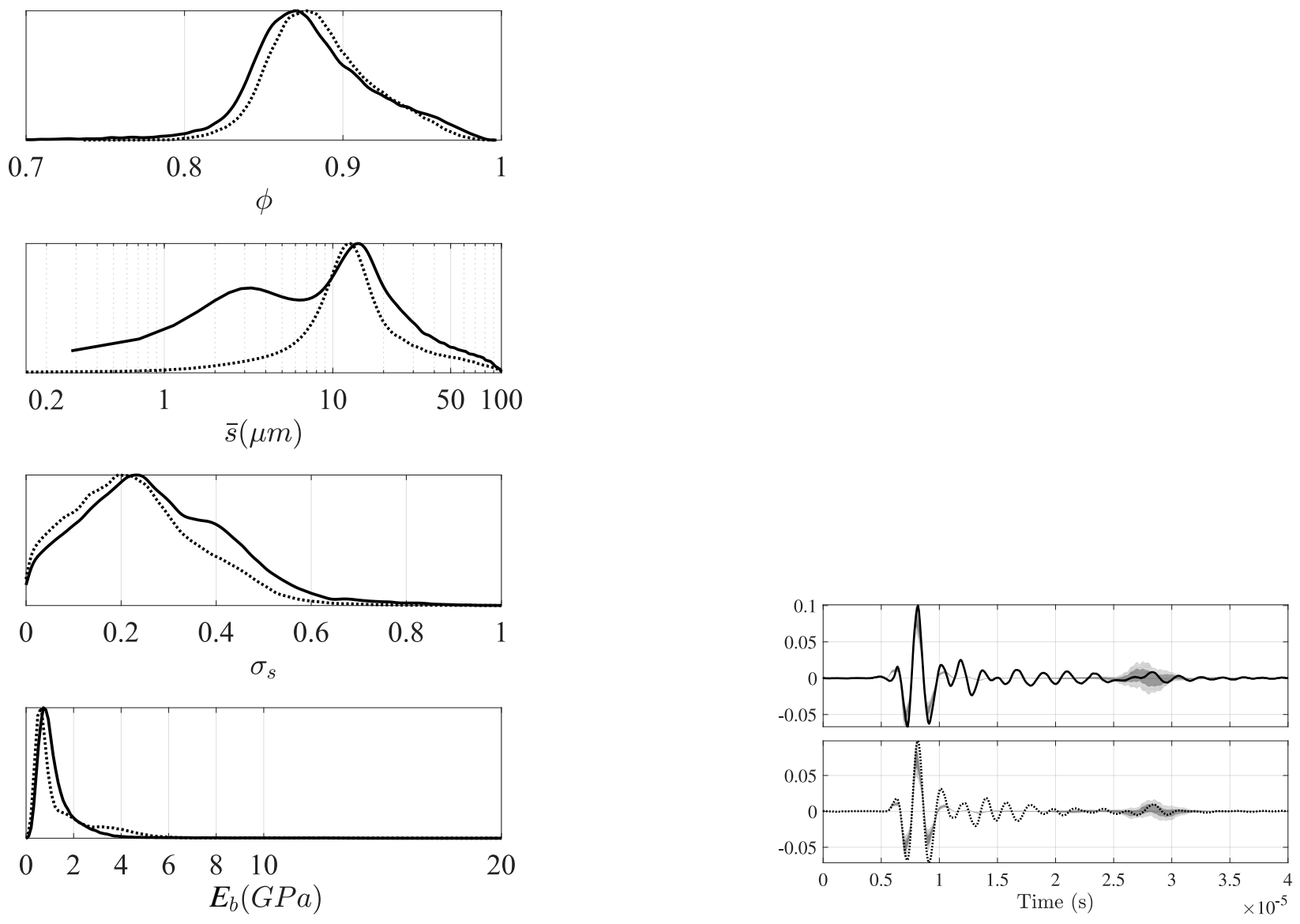

(a) Probability density functions of selected bone (b) Experiment vs fitted model comparison of time structural parameters for different measurements domain reflected signals on the bone sample.

Figure 6: Inference results for sample M4 

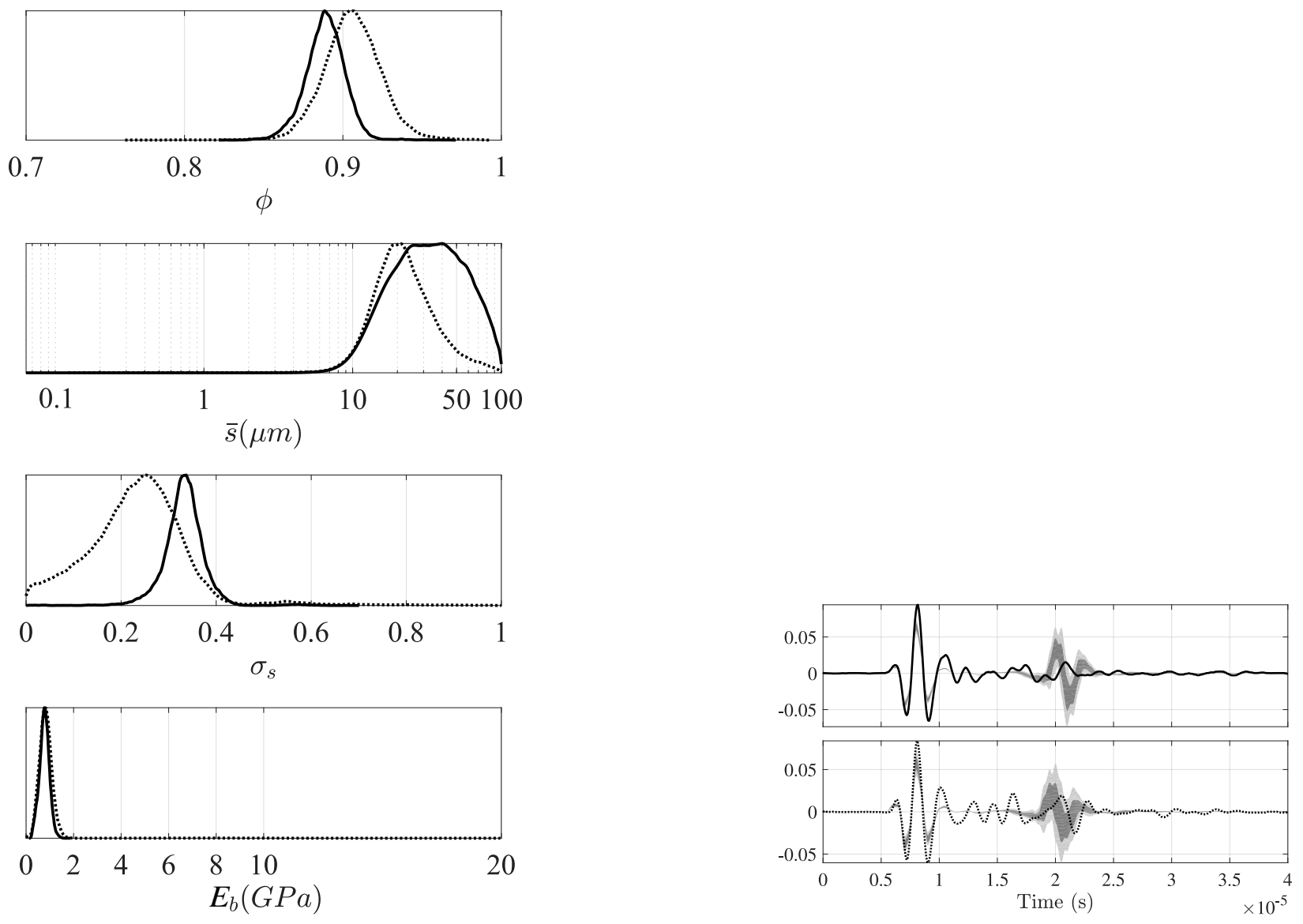

(a) Probability density functions of selected bone (b) Experiment vs fitted model comparison of time structural parameters for different measurements domain reflected signals on the bone sample.

Figure 7: Inference results for sample M5 

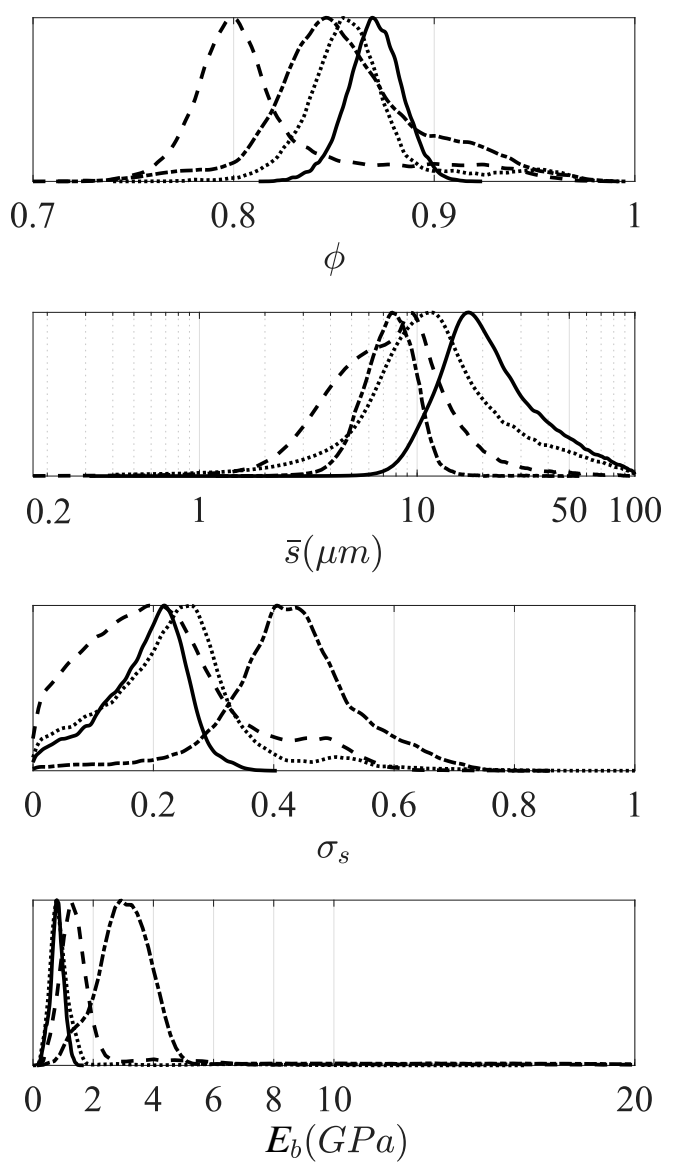

(a) Probability density functions of selected bone structural parameters for different measurements on the bone sample.

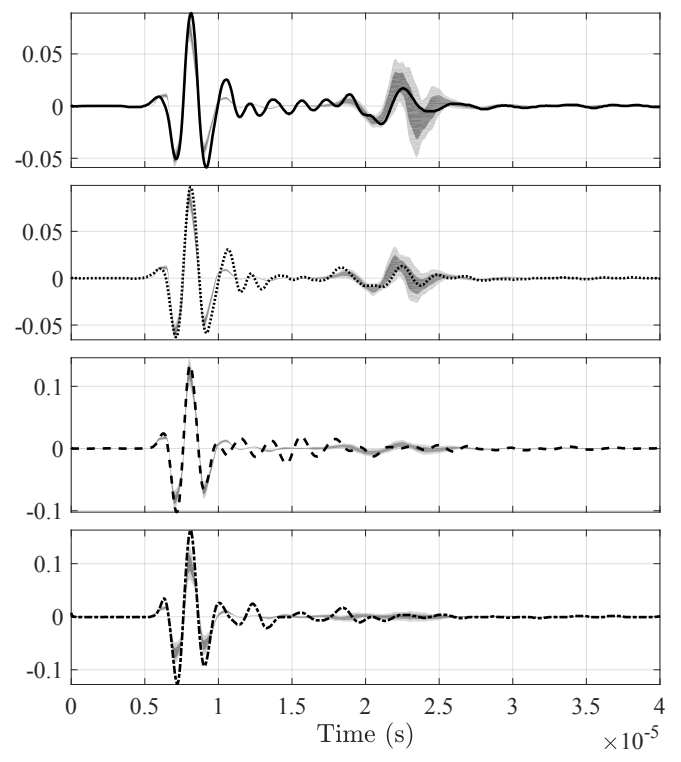

(b) Experiment vs fitted model comparison of time domain reflected signals

Figure 8: Inference results for sample M6 

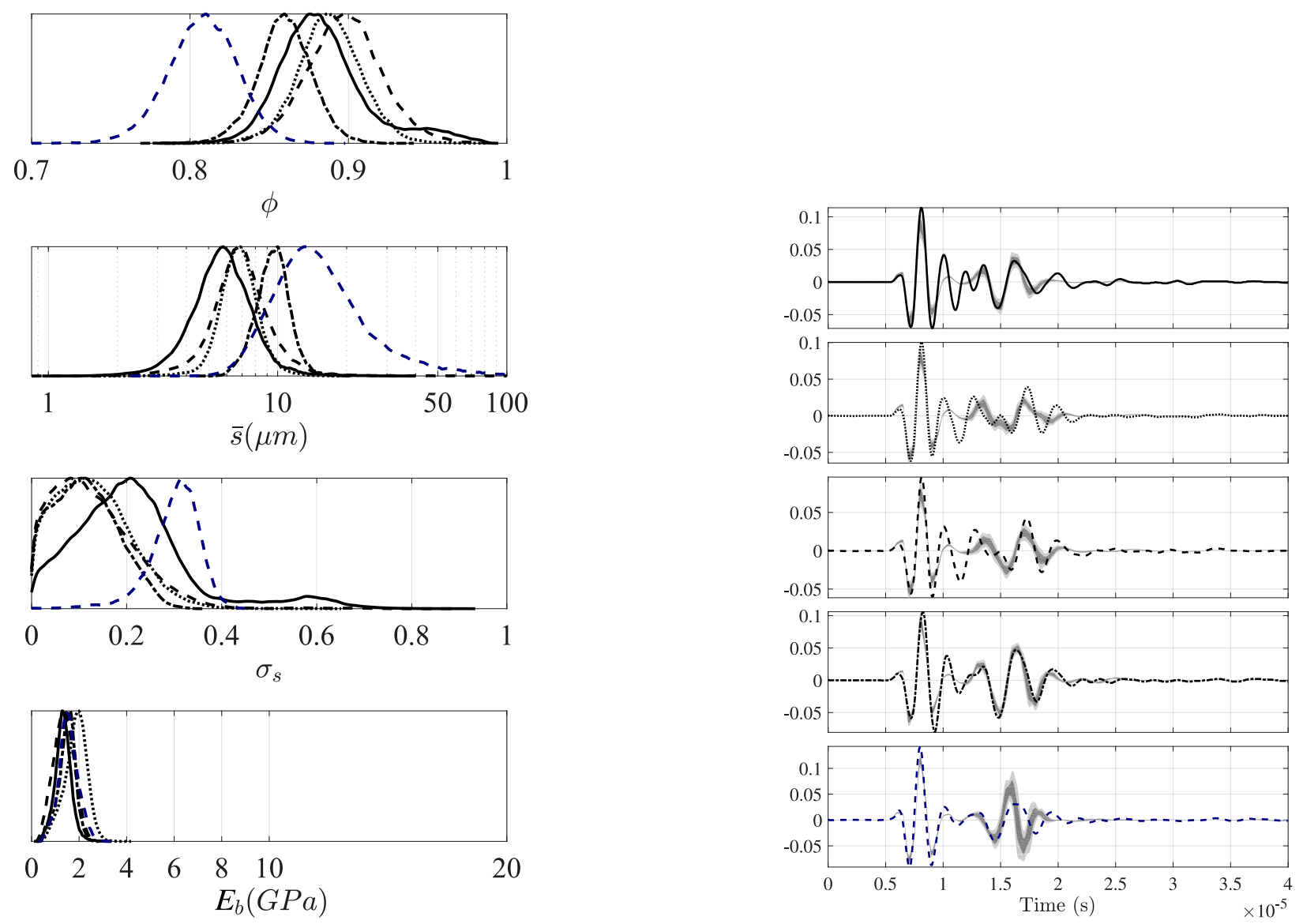

(a) Probability density functions of selected bone structural parameters for different measurements on the bone sample.

Figure 9: Inference results for sample M7 


\section{Discussion}

The inference results obtained in this work are overall consistent with what could be expected for bone samples from femoral heads. One of the limitations of the present work is the lack of comparison between the identified properties and those directly measured on these samples - comparison which could be obtained with dedicated equipment that we are currently lacking. Comparisons with what can be found in the literature are not always insightful, given the very large spread of measured values.

The use of the Horoshenkov model proved adequate for the task at hand. The model seems able to capture well the main features of the reflected ultrasonic signals within the bone samples (good fit between experimental and numerical reflected signals) and also seems robust enough, in combination with the selected Bayesian inference framework, to identify material properties. It was quite satisfying to observe similar parameter pdfs across different samples or different test sites on the same sample. It seems that for the subset of parameters of interest selected in this work (porosity, pore mean size and standard deviation, and Young modulus), the problem is well-posed. However, reflected signals alone might not be sufficient to fully characterize bone samples. In particular, it was not possible to identify $\rho_{s}$, the solid bone tissue density, an important parameter of bone strength.

A natural extension of this work would be to increase the complexity of the Horoshenkov model in order to identify the parameters pertaining to the definition of the pore shape.

Another potential extension of this work would be to develop prior modeling for some intrinsic properties, based on different experiments. An experiment that could be used is the ultrasonic backscattering measurement, which also feeds on reflected ultrasonic signals, albeit at higher frequencies, where diffusion effects predominate within the bone sample. In this experiment, some bone properties could be inferred, thus providing a prior density function for the method presented in this work.

A difficult but necessary extension of this work (towards in vivo testing) would be the consideration of heterogeneity in the sample. While the direct problem of wave propagation in these kind of material has already been devised (see e.g., Ref. [36]), studies on Bayesian inference on this type of problem are lacking. Further including the cortical bone, muscle and skin elements in the modeling (as done in Ref. [47) could then potentially lead to in vivo applications. 


\section{Conclusion}

This article has introduced a general identification method for bone samples, based on ultrasonic signals reflected through bone samples saturated by water. A statistical inference strategy was used to identify the microstructural and mechanical properties of seven porous samples. The direct problem consisted in the Biot equations of wave propagation inside a poroelastic material coupled with the Horoshenkov model to account for the wave dissipation within the pores. The advantage of this statistical inference method was that it provides an easy interpretation of the results, in the form of probability density functions of the model parameters, and a robust way to take into account prior knowledge on the different identified properties, if any.

The method robustness was tested by repeating the inference process on different signals obtained on the same samples. Some parameters were not well identified, because of their lack of sensitivity relative to the reflection coefficient. Still, the main parameters of interest were identified (porosity, mean pore size and frame Young modulus), displaying well defined probability density functions, and values within the expected range for femoral head samples.

\section{Acknowledgment}

This work has been conducted under the ONERA-CNRS collaboration agreement on porous media acoustics. The authors would like to thank Estelle Piot for her comments on the initial version of this work.

\section{References}

[1] R. Marcus, D. Feldman, D. W. Dempster, M. Luckey, J. A. Cauley, Osteoporosis, 4th Edition, Elsevier Science, 2013. doi:10.1016/C2011-1-04566-6.

[2] C.-C. Glüer, I. Q. U. C. Group, Quantitative ultrasound techniques for the assessment of osteoporosis: expert agreement on current status, Journal of Bone and Mineral Research 12 (8) (1997) 1280-1288. doi:10.1359/jbmr.1997.12.8.1280

[3] C. Langton, S. Palmer, R. Porter, The measurement of broadband ultrasonic attenuation in cancellous bone, Engineering in medicine 13 (2) (1984) 89-91. doi:10.1243/EMED_JOUR_1984_013_022_02. 
[4] M. McKelvie, S. Palmer, The interaction of ultrasound with cancellous bone, Physics in Medicine \& Biology 36 (10) (1991) 1331. doi:10.1088/0031-9155/36/10/003.

[5] J. L. Williams, Ultrasonic wave propagation in cancellous and cortical bone: Prediction of some experimental results by biot's theory, The Journal of the Acoustical Society of America 91 (2) (1992) 1106-1112. doi:10.1121/1.402637.

[6] C. F. Njeh, The dependence of ultrasound velocity and attenuation on the material properties of cancellous bone, Ph.D. thesis, Sheffield Hallam University, (1995).

[7] J. Williams, M. Grimm, F. Wehrli, K. Foster, H. Chung, Prediction of frequency and pore size dependent attenuation of ultrasound in trabecular bone using biot's theory, in: Mechanics of Poroelastic Media, Springer, 1996, pp. 263-271. doi:10.1007/978-94-015-8698-6_15.

[8] R. Hodgskinson, C. Njeh, M. Whitehead, C. Langton, The non-linear relationship between bua and porosity in cancellous bone, Physics in Medicine \& Biology 41 (11) (1996) 2411. doi:10.1088/ $0031-9155 / 41 / 11 / 012$

[9] A. Hosokawa, T. Otani, Ultrasonic wave propagation in bovine cancellous bone, The Journal of the Acoustical Society of America 101 (1) (1997) 558-562. doi:10.1121/1.418118.

[10] K. A. Wear, B. S. Garra, Assessment of bone density using ultrasonic backscatter, Ultrasound in medicine \& biology 24 (5) (1998) 689-695. doi:10.1016/S0301-5629(98)00040-4

[11] K. A. Wear, Frequency dependence of ultrasonic backscatter from human trabecular bone: Theory and experiment, The Journal of the Acoustical Society of America 106 (6) (1999) 3659-3664. doi: $10.1121 / 1.428218$

[12] K. A. Wear, A. P. Stuber, J. C. Reynolds, Relationships of ultrasonic backscatter with ultrasonic attenuation, sound speed and bone mineral density in human calcaneus, Ultrasound in medicine \& biology 26 (8) (2000) 1311-1316. doi:10.1016/S0301-5629(00)00267-2.

[13] L. Cardoso, F. Teboul, L. Sedel, C. Oddou, A. Meunier, In vitro acoustic waves propagation in human and bovine cancellous bone, Journal of Bone and Mineral Research 18 (10) (2003) 1803-1812. doi: $10.1359 / \mathrm{j} \mathrm{bmr.2003.18.10.1803.}$ 
[14] Z. E. A. Fellah, J. Y. Chapelon, S. Berger, W. Lauriks, C. Depollier, Ultrasonic wave propagation in human cancellous bone: Application of biot theory, The Journal of the Acoustical Society of America 116 (1) (2004) 61-73. doi:10.1121/1.1755239.

[15] K. A. Wear, T. A. Stiles, G. R. Frank, E. L. Madsen, F. Cheng, E. J. Feleppa, C. S. Hall, B. S. Kim, P. Lee, W. D. O’Brien Jr, et al., Interlaboratory comparison of ultrasonic backscatter coefficient measurements from 2 to $9 \mathrm{mhz}$, Journal of ultrasound in medicine 24 (9) (2005) 1235-1250. doi: 10.7863/jum.2005.24.9.1235.

[16] P. Laugier, G. Haïat, Bone quantitative ultrasound, Vol. 576, Springer, 2011. doi:10.1007/ 978-94-007-0017-8

[17] C. Njeh, C. Boivin, C. Langton, The role of ultrasound in the assessment of osteoporosis: a review, Osteoporosis international 7 (1) (1997) 7-22. doi:10.1007/BF01623454.

[18] K. R. Marutyan, M. R. Holland, J. G. Miller, Anomalous negative dispersion in bone can result from the interference of fast and slow waves, The Journal of the Acoustical Society of America 120 (5) (2006) EL55-EL61. doi:10.1121/1.2357187.

[19] E. R. Hughes, T. G. Leighton, P. R. White, G. W. Petley, Investigation of an anisotropic tortuosity in a biot model of ultrasonic propagation in cancellous bone, The Journal of the Acoustical Society of America 121 (1) (2007) 568-574. doi:10.1121/1.2387132.

[20] M. Pakula, F. Padilla, P. Laugier, M. Kaczmarek, Application of biot's theory to ultrasonic characterization of human cancellous bones: determination of structural, material, and mechanical properties, The Journal of the acoustical Society of america 123 (4) (2008) 2415-2423. doi:10.1121/1.2839016.

[21] G. Rus, M. Pakula, Q. Grimal, P. Laugier, Information theory framework to reconstruct biot constants of trabecular bone from ultrasound, in: 2015 6th European Symposium on Ultrasonic Characterization of Bone, 2015, pp. 1-4. doi:10.1109/ESUCB.2015.7169901.

[22] H. Chen, R. P. Gilbert, P. Guyenne, A biot model for the determination of material parameters of cancellous bone from acoustic measurements, Inverse Problems 34 (8) (2018) 085009. doi:10.1088/ $1361-6420 /$ aac520.

[23] J.-D. Chazot, E. Zhang, J. Antoni, Acoustical and mechanical characterization of poroelastic materials using a bayesian approach, J. Acoust. Soc. Am. 131 (6) (2012) 4584-4595. doi:10.1121/1.3699236 
[24] M. Niskanen, O. Dazel, J.-P. Groby, A. Duclos, T. Lähivaara, Characterising poroelastic materials in the ultrasonic range-a bayesian approach, Journal of Sound and Vibration 456 (2019) 30-48. doi: $10.1016 / j \cdot j$ sv.2019.05.026

[25] R. Roncen, Z. Fellah, E. Piot, E. Ogam, Bayesian inference of a human bone and biomaterials using ultrasonic transmitted signals, The Journal of the Acoustical Society of America 146 (3) (2019) 16291640. doi:10.1121/1.5125263

[26] J. Karjalainen, O. Riekkinen, J. Töyräs, M. Hakulinen, H. Kröger, T. Rikkonen, K. Salovaara, J. Jurvelin, Multi-site bone ultrasound measurements in elderly women with and without previous hip fractures, Osteoporosis international 23 (4) (2012) 1287-1295. doi:10.1007/s00198-011-1682-2.

[27] B. K. Hoffmeister, A. R. Wilson, M. J. Gilbert, M. E. Sellers, A backscatter difference technique for ultrasonic bone assessment, The Journal of the Acoustical Society of America 132 (6) (2012) 4069-4076. doi:10.1121/1.4763992

[28] M. Malo, J. Töyräs, J. Karjalainen, H. Isaksson, O. Riekkinen, J. Jurvelin, Ultrasound backscatter measurements of intact human proximal femurs-relationships of ultrasound parameters with tissue structure and mineral density, Bone 64 (2014) 240-245. doi:10.1016/j.bone.2014.04.014.

[29] Z. E. A. Fellah, S. Berger, W. Lauriks, C. Depollier, C. Aristegui, J.-Y. Chapelon, Measuring the porosity and the tortuosity of porous materials via reflected waves at oblique incidence, J. Acoust. Soc. Am. 113 (5) (2003) 2424-2433. doi:10.1121/1.1567275.

[30] Z. E. A. Fellah, F. Mitri, C. Depollier, S. Berger, W. Lauriks, J. Chapelon, Characterization of porous materials with a rigid frame via reflected waves, J. Appl. Acoust. 94 (12) (2003) 7914-7922. doi: $10.1063 / 1.1629386$

[31] Z. E. A. Fellah, S. Berger, W. Lauriks, C. Depollier, P. Trompette, J. Chapelon, Ultrasonic measurement of the porosity and tortuosity of air-saturated random packings of beads, J. Appl. Acoust. 93 (11) (2003) 9352-9359. doi:10.1063/1.1572191.

[32] R. Roncen, Z. E. A. Fellah, F. Simon, E. Piot, M. Fellah, E. Ogam, C. Depollier, Bayesian inference for the ultrasonic characterization of rigid porous materials using reflected waves by the first interface, The Journal of the Acoustical Society of America 144 (1) (2018) 210-221. doi:10.1121/1.5044423. 
[33] K. Horoshenkov, M. Swift, The acoustic properties of granular materials with pore size distribution close to log-normal, The Journal of the Acoustical Society of America 110 (5) (2001) 2371-2378. doi: $10.1121 / 1.1408312$

[34] D. L. Johnson, J. Koplik, R. Dashen, Theory of dynamic permeability and tortuosity in fluid-saturated porous media, J. Fluid Mech. 176 (1987) 379-402. doi:10.1017/S0022112087000727.

[35] M. A. Biot, Mechanics of deformation and acoustic propagation in porous media, J. Appl. Acoust. 33 (4) (1962) 1482-1498. doi:10.1063/1.1728759.

[36] G. Gautier, L. Kelders, J.-P. Groby, O. Dazel, L. De Ryck, P. Leclaire, Propagation of acoustic waves in a one-dimensional macroscopically inhomogeneous poroelastic material, The Journal of the Acoustical Society of America 130 (3) (2011) 1390-1398. doi:10.1121/1.3605530

[37] O. Dazel, J.-P. Groby, B. Brouard, C. Potel, A stable method to model the acoustic response of multilayered structures, Journal of Applied Physics 113 (8) (2013) 083506. doi:10.1063/1.4790629.

[38] K. V. Horoshenkov, K. Attenborough, S. Chandler-Wilde, Padé approximants for the acoustical properties of rigid frame porous media with pore size distributions, The journal of the acoustical society of America 104 (3) (1998) 1198-1209. doi:10.1121/1.424328.

[39] T. Doktor, J. Valach, D. Kytỳr, O. Jiroušek, Pore size distribution of human trabecular bone: Comparison of intrusion measurements with image analysis, Engineering Mechanics.

[40] K. V. Horoshenkov, A. Hurrell, J.-P. Groby, A three-parameter analytical model for the acoustical properties of porous media, The Journal of the Acoustical Society of America 145 (4) (2019) 2512-2517. doi:10.1121/1.5098778

[41] K. V. Horoshenkov, A. Hurrell, J.-P. Groby, Erratum: A three-parameter analytical model for the acoustical properties of porous media [j. acoust. soc. am. 145 (4), 2512-2517 (2019)], The Journal of the Acoustical Society of America 147 (1) (2020) 146-146. doi:10.1121/10.0000560.

[42] S. B. Lang, Ultrasonic method for measuring elastic coefficients of bone and results on fresh and dried bovine bones, IEEE Transactions on Biomedical Engineering (2) (1970) 101-105. doi:10.1109/TBME. 1970.4502706 .

[43] R. C. Smith, Uncertainty quantification: theory, implementation, and applications, Vol. 12, Siam, Philadelphia, 2013. 
[44] S. R. Pride, F. D. Morgan, A. F. Gangi, Drag forces of porous-medium acoustics, Phys. Rev. B 47 (9) (1993) 4964-4978. doi:10.1103/PhysRevB.47.4964.

[45] E. Laloy, J. A. Vrugt, High-dimensional posterior exploration of hydrologic models using multipletry dream(zs) and high-performance computing, Water Resour. Res. 48 (1) (2012) n/a-n/a, w01526. doi:10.1029/2011WR010608,

[46] R. Hodgskinson, J. Currey, Young's modulus, density and material properties in cancellous bone over a large density range, Journal of Materials Science: Materials in Medicine 3 (5) (1992) 377-381. doi: 10.1007/BF00705371.

[47] R. P. Gilbert, Y. Liu, J.-P. Groby, E. Ogam, A. Wirgin, Y. Xu, Computing porosity of cancellous bone using ultrasonic waves, ii: The muscle, cortical, cancellous bone system, Mathematical and computer modelling 50 (3-4) (2009) 421-429. doi:10.1016/j.mcm.2008.06.021. 
Table 3: Inference results, mean and standard deviation. The "a.b" notation of the left column indicates both the femoral head sample "a" and the number of the test site location "b"

\begin{tabular}{|c|c|c|c|c|c|c|c|c|}
\hline Sample & $\phi$ & $\bar{s}$ & $\sigma_{s}$ & $K_{s}$ & $\xi_{s}$ & $E_{b}$ & $\xi_{b}$ & $\nu_{b}$ \\
\hline & - & $\mu \mathrm{m}$ & - & $\mathrm{GPa}$ & - & $\mathrm{GPa}$ & - & - \\
\hline 1.1 & $0.89 \pm 0.01$ & $5.4 \pm 0.5$ & $0.13 \pm 0.07$ & $32.2 \pm 26.8$ & $0.55 \pm 0.21$ & $1.84 \pm 0.41$ & $0.08 \pm 0.05$ & $0.22 \pm 0.10$ \\
\hline 1.2 & $0.95 \pm 0.02$ & $4.2 \pm 0.8$ & $0.14 \pm 0.08$ & $65.8 \pm 35.8$ & $0.48 \pm 0.22$ & $1.85 \pm 0.42$ & $0.12 \pm 0.08$ & $0.22 \pm 0.10$ \\
\hline 2.1 & $0.78 \pm 0.01$ & $6.0 \pm 0.8$ & $0.11 \pm 0.07$ & $46.7 \pm 33.0$ & $0.47 \pm 0.23$ & $\begin{array}{l}2.59 \pm 0.65 \\
6.45 \pm 1.03\end{array}$ & $0.59 \pm 0.30$ & $0.20 \pm 0.10$ \\
\hline 2.2 & $0.86 \pm 0.03$ & $6.9 \pm 1.4$ & $0.18 \pm 0.08$ & $82.6 \pm 40.1$ & $0.50 \pm 0.23$ & $\begin{array}{l}2.41 \pm 0.73 \\
13.5 \pm 2.58\end{array}$ & $0.54 \pm 0.23$ & $0.20 \pm 0.09$ \\
\hline 2.3 & $0.85 \pm 0.02$ & $16.8 \pm 16.1$ & $0.22 \pm 0.12$ & $55.0 \pm 37.8$ & $0.46 \pm 0.22$ & $1.86 \pm 0.57$ & $0.36 \pm 0.18$ & $0.23 \pm 0.09$ \\
\hline 3.1 & $0.90 \pm 0.02$ & $23.2 \pm 18.5$ & $0.28 \pm 0.11$ & $56.6 \pm 38.9$ & $0.45 \pm 0.22$ & $1.17 \pm 0.39$ & $0.30 \pm 0.15$ & $0.22 \pm 0.10$ \\
\hline 3.2 & $0.86 \pm 0.01$ & $8.2 \pm 1.5$ & $0.09 \pm 0.06$ & $50.5 \pm 38.0$ & $0.43 \pm 0.22$ & $1.98 \pm 0.45$ & $0.11 \pm 0.06$ & $0.23 \pm 0.10$ \\
\hline 4.1 & $0.88 \pm 0.05$ & $29.2 \pm 22.8$ & $0.27 \pm 0.15$ & $45.4 \pm 36.9$ & $0.46 \pm 0.22$ & $1.36 \pm 1.41$ & $0.58 \pm 0.16$ & $0.23 \pm 0.09$ \\
\hline 4.2 & $0.89 \pm 0.03$ & $28.6 \pm 22$ & $0.23 \pm 0.13$ & $55.5 \pm 37.0$ & $0.45 \pm 0.23$ & $1.59 \pm 1.74$ & $0.53 \pm 0.19$ & $0.23 \pm 0.10$ \\
\hline 5.1 & $0.89 \pm 0.01$ & $48.1 \pm 21.7$ & $0.33 \pm 0.05$ & $44.8 \pm 36.6$ & $0.46 \pm 0.22$ & $0.77 \pm 0.21$ & $0.65 \pm 0.12$ & $0.24 \pm 0.10$ \\
\hline 5.2 & $0.91 \pm 0.02$ & $31.9 \pm 17.8$ & $0.23 \pm 0.11$ & $49.9 \pm 39.3$ & $0.46 \pm 0.22$ & $0.89 \pm 0.76$ & $0.64 \pm 0.17$ & $0.24 \pm 0.10$ \\
\hline 6.1 & $0.87 \pm 0.01$ & $34.0 \pm 20$ & $0.18 \pm 0.07$ & $44.6 \pm 36.3$ & $0.47 \pm 0.22$ & $0.80 \pm 0.21$ & $0.37 \pm 0.12$ & $0.23 \pm 0.10$ \\
\hline 6.2 & $0.86 \pm 0.03$ & $27.8 \pm 21.5$ & $0.23 \pm 0.12$ & $42.3 \pm 39.2$ & $0.47 \pm 0.22$ & $1.15 \pm 1.31$ & $0.46 \pm 0.18$ & $0.23 \pm 0.10$ \\
\hline 6.3 & $0.82 \pm 0.05$ & $14.2 \pm 12.8$ & $0.21 \pm 0.13$ & $54.0 \pm 41.0$ & $0.44 \pm 0.22$ & $2.78 \pm 4.47$ & $0.44 \pm 0.16$ & $0.23 \pm 0.09$ \\
\hline 6.4 & $0.86 \pm 0.04$ & $8.4 \pm 3.0$ & $0.43 \pm 0.11$ & $60.4 \pm 39.0$ & $0.47 \pm 0.22$ & $3.99 \pm 3.89$ & $0.36 \pm 0.13$ & $0.22 \pm 0.10$ \\
\hline 7.1 & $0.88 \pm 0.03$ & $6.7 \pm 2.7$ & $0.21 \pm 0.13$ & $45.0 \pm 36.1$ & $0.47 \pm 0.22$ & $1.33 \pm 0.34$ & $0.29 \pm 0.11$ & $0.23 \pm 0.10$ \\
\hline 7.2 & $0.89 \pm 0.02$ & $7.2 \pm 1.5$ & $0.13 \pm 0.08$ & $54.4 \pm 39.1$ & $0.46 \pm 0.21$ & $1.86 \pm 0.47$ & $0.11 \pm 0.1$ & $0.23 \pm 0.09$ \\
\hline 7.3 & $0.90 \pm 0.02$ & $8.6 \pm 6.7$ & $0.13 \pm 0.09$ & $50.7 \pm 37.6$ & $0.45 \pm 0.22$ & $1.35 \pm 0.43$ & $0.22 \pm 0.17$ & $0.24 \pm 0.01$ \\
\hline 7.4 & $0.86 \pm 0.02$ & $9.9 \pm 1.7$ & $0.11 \pm 0.07$ & $51.7 \pm 37.5$ & $0.46 \pm 0.22$ & $1.48 \pm 0.36$ & $0.17 \pm 0.06$ & $0.23 \pm 0.10$ \\
\hline 7.5 & $0.81 \pm 0.02$ & $24.2 \pm 16.8$ & $0.30 \pm 0.05$ & $37.9 \pm 34.6$ & $0.52 \pm 0.22$ & $1.56 \pm 0.41$ & $0.56 \pm 0.15$ & $0.21 \pm 0.10$ \\
\hline
\end{tabular}

